\title{
Coral Reef Image Classification Employing Deep Features and A Novel Local Inter Cross Weber Magnitude (LICWM) Pattern
}

\author{
C.Padma Priya ${ }^{1}$, Dr.S.Muruganantham ${ }^{2}$ \\ ${ }^{1}$ Research Scholar in Computer Science (18223152162024) - ST.HinduCollege, Nagercoil. \\ ${ }^{2}$ Associate Professor of Computer Science - S T.HinduCollege,Nagercoil. \\ Affiliated to Manonmanim Sundaranar University, \\ Tirunelveli-627412.
}

Article History:Received:11 november 2020; Accepted: 27 December 2020; Published online: 05 April 2021 ABSTRACT : Coral reefs are essential in marine ecosystem as they sustain a great part of marine life. The
automatic classification of corals on submarine images is so much important in recent times. Hence, it can assist
marine experts to classify endangered and susceptible coral reefs. But, classifying coral reef images is a
promising task due to its varying color, texture, shape and morphology. The main objective of this work is to
propose a novel operator called Local Inter Cross Weber Magnitude (LICWM) pattern. For classification,
VGG-16 architecture is applied for extracting the features of coral reef images. VGG- 16 architecture has many
layers for extracting deep features effectively. The traditional methods used K-Nearest Neighbor (KNN) and
Random Forest (RF) for classifying deep features. The performance of the proposed method is estimated using
F-score. The Experimental results show that the proposed operator achieves better accuracy level and
performance with EILAT and RSMAS data sets.

Keywords: VGG, KNN, Random Forest, Coral reef, classification

\section{INTRODUCTION}

Coral reefs are gorgeous and dynamic ecosystems providing habitat to one quarter of all marine species. They are capable in service with an annual commercial value of billions of dollars to humans each year. Coral reefs are aquatic habitat on which numerous ocean species depend on them for food and shelter. They found in tropical and subtropical nations. Coral reefs are also called as the rain forest of ocean. The amazing colors of coral reefs are due to zooxanthellae algae which live inside coral polyps. Coral reefs are very important to people for their livelihood and crucial economic opportunity. It also protects coastlines from life threatening storms.

Nowadays, coral reefs are under threat due to climatic change, unsustainable coastal development, pollution and destructive fishing practices etc., Coral bleaching is occurred by global warming. Unfortunately, many species of coral are destroyed. There is a need to conserve and to preserve coral reef. Many scientists analyses and monitors coral reef using several techniques depending on their similarities, texture and color. The classification of coral reef images is a great challenge due to its impurity, blurred images and class variations. Many Machine learning algorithms are also in use to classify coral reef images according to their classes.

The motivation of this work is to extract and to classify coral reef images according to their classes. This work extracts and classify coral reef images using VGG-16 embedded with novel descriptor named Local Inter Cross Weber Magnitude Pattern.

The main work are the following:

$>$ The features of coral reef images are extracted using a novel operator called Local Inter Cross Weber Magnitude (LICWM) and classified using KNN and Random Forest classifiers.

$>$ The results of the proposed operator with VGG-16 network are used for extracting deep features.

$>$ Experiments are conducted again by embedding the handcrafted features of LICWM with deep features of VGG and the results are analysed.

$>$ The results are compared based on their classification with various descriptors.

\section{RELATED WORKS}

There are certain works for representing coral reef image classification. Jiechen et al [1] have proposed Weber Local Descriptor based on Webers Law to compute a histogram by encoding differential excitation and orientation. WLD computation implementation is faster than SIFT and LBP operators. Joshua et al [2] have presented a novel technique for coral reef image segmentation. Scale Invariant Feature Transform (SIFT) is used for object recognition and SVM is used as classifier. Harsan Dawood et al [3] have proposed Weber Local Descriptor for classifying the texture of an image. This method provides significant improvement than the other state of art methods, like Local Binary Pattern (LBP), Local Phase Quantization (LPQ) and Weber Local Descriptor (WLD). Deepshika et al [4] have implemented Webers law based LBP with Center pixel (WLBPC)

*Corresponding author: C.Padma Priya ${ }^{1}$

${ }^{1}$ Research Scholar in Computer Science (18223152162024) - ST.HinduCollege, Nagercoil 
due to its robustness and discriminating capabilities. Agarwal et al [5] have investigated Webers native Descriptor (WLD) to recognize gender. Minimum Distance Measurement and neural network is used for classification. Amit Satpathy et al [6] have proposed Discriminative Robust Local Binary Pattern (DRLBP) and Discriminative Robust Local Ternary pattern (DRLTP) for object recognition. It solves discrimination between light object backgrounds against dark background.

Jinwang Feng et al [7] have extracted an image features using dominant Complemented modelling of the traditional Local Binary Pattern (LBP) operator. It gives best results than the other LBP variants. Guo et al [8] have proposed Completed LBP (CLBP) with three operators, namely CLBP_C, CLBP_S and CLBP_M for texture classification. Chang Pei et al [9] have implemented two descriptors such as LBP and Weber Local Binary Pattern (WLBP) to recognize gray scale images, depth images and 2D + depth images. The processing time is improved with 90\% accuracy. Ahila et al [10] have extracted the features of an image by WLD which is the composition of differential excitation and differential orientation. SVM based classifiers are used for classification. Fan Liu et al [11] have presented Weber Local Binary Pattern (WLBP) which is the combination of WLD and LBP. The future work is to reduce feature vector and to improve the computing efficiency. Zuodong Yang et al [12] have proposed Weber Local Pattern (WLP) and Weber Ternary Pattern (WTP) based on Weber's law for evaluating local gray scale difference. It reveals good scale difference. Thanh Phuong Ngugen et al [13] have presented a texture framework called Statistical Binary Patterns (SBP) by applying rotation invariant binary patterns to images. Ngoc-Son Vu et al [14] have used Patterns of Orientation Difference (POD) for exploring relationships between gradient orientation and magnitude for various image structures. Here, the whitened Principal Component Analysis (PCA) is used as Descriptors.

Mohammad Hossein Shakoori et al [15] have proposed Extended Completed Local Binary Pattern (CLBP) for feature extraction. Ani Brown Mary et al [16] have proposed Octa-angled Pattern for Triangular sub region (OPT) for extracting features of pixel in clockwise directions from triangular pattern of its neighbor. For further classification Pulse Coupled Convolutional Neural Network (PCCNN) is also employed. Ani Brown Mary et al [17] have proposed a novel feature descriptor named Z with Tilted Z local Binary Pattern for coral reef image feature extraction and classification. It is an accurate and efficient feature descriptor for reducing the size of feature vector. Also, Ani Brown Mary et al [18] have implemented Improved Local Derivative Pattern (ILBP) for coral reef image classification. While comparing with other classification methods it achieves highest accuracy. Hatibaruah [19] has introduced novel feature descriptor called Elliptical Local Binary Co-occurrence Pattern (EBCOP). It is the combination of sparse Elliptical Local Binary Pattern (ELBP) and Grey Level Cooccurrence matrix (GLCM). It shows significant improvement than other feature descriptors. Ani Brown Mary et al [20] have also implemented feature descriptor and machine learning techniques to classify the diseased images of coral reef. Padma Priya [21] has classified coral reef images with hybrid methods of traditional methods with deep learning. In that analysis IWBC achieves best performance than other traditional methods of LBP and LAP. Padma Priya [22] has also proposed VGG 16 deep layered architecture for feature extraction of coral reef image. It achieves best performance than LBP and LAP techniques.

\section{METHODOLOGY}

The methodology implemented in this proposed method is a novel operator, namely Local Inter Cross Weber Magnitude Pattern derived from Improved Weber's Local Descriptor (IWLD) which is based on Weber's Law. It states that the change can only be identified if the rate of the change of stimulus to original stimulus is high enough. According to Weber's law, two components are used to determine the texture of the images with differential excitation and orientation. To avoid the discrimination between the pixels of an image, the proposed operator is introduced. It is an efficient, robust, and discriminative feature descriptor. VGG net architecture is also integrated with this proposed operator to extract deep features of coral reef image and to classify one class from another. It gives better result with its hybrid architecture which is depicted in the Figure 1.

Two data sets which are used for experiments are RSMAS and EILAT. Figure 1 shows that the input images are extracted using the hybrid methods of the proposed operator with deep learning method. VGG-16 is used for deep learning which extracts the features effectively when combined with the proposed operator LICWMP. KNN and Random Forest is used for classification purposes after feature extraction. 


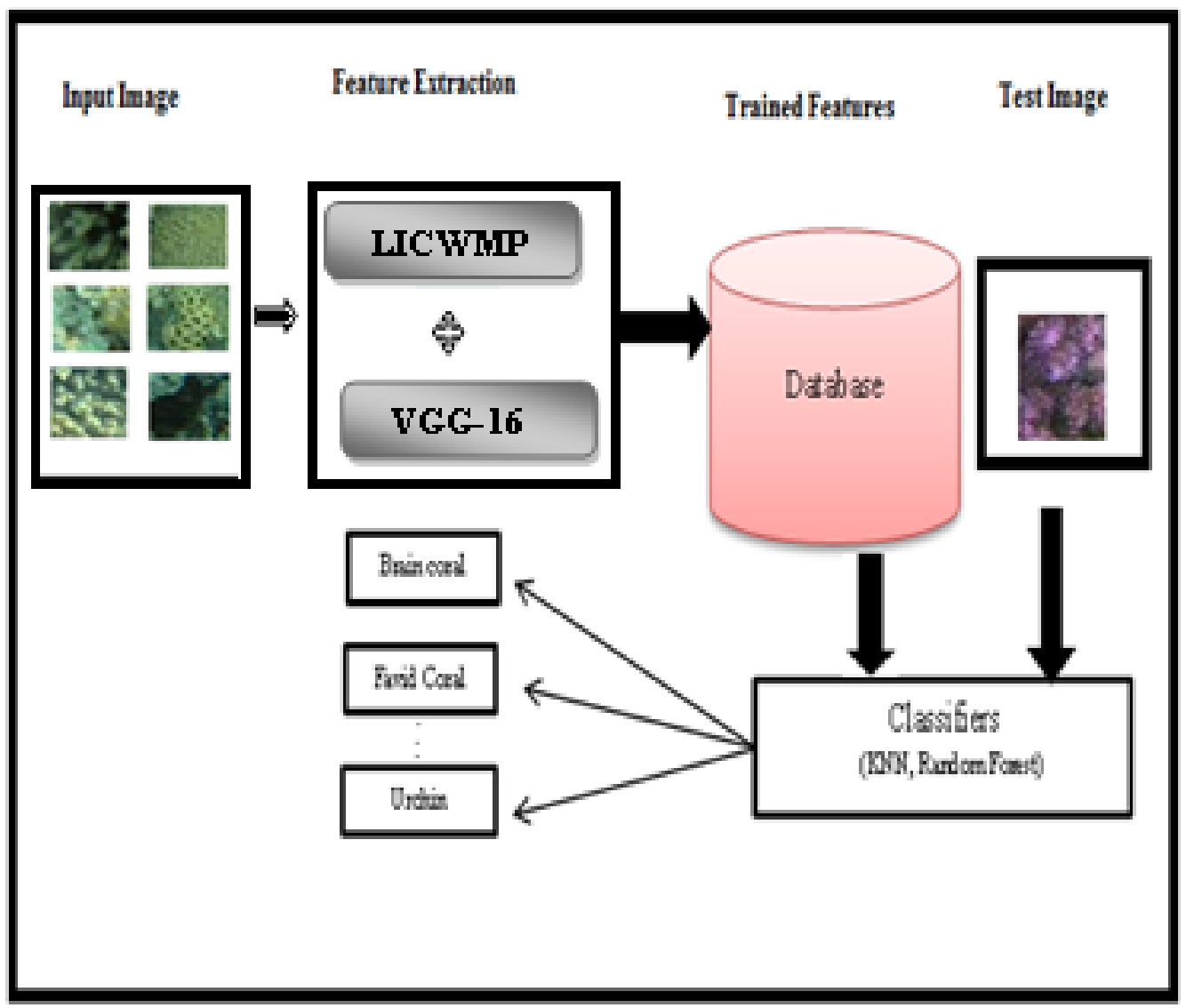

Figure 1 Methodology for coral reef image classification

The proposed operator is developed by the determination of Magnitude Patterns from the existing Improved Weber Local Descriptor. The Magnitude Patterns are derived from the pixel and its eight neighbors with corresponding directions, which is depicted in the Figure 2.

\begin{tabular}{|c|c|c|}
\hline$X_{0}$ & $X_{1}$ & $X_{2}$ \\
\hline$X_{7}$ & $X_{C}$ & $X_{3}$ \\
\hline$X_{6}$ & $X_{5_{2}}$ & $X_{4}$ \\
\hline
\end{tabular}

Figure 2 Representation of a coral reef image with its pixels and its neighbors 3.1 Determination of Magnitude Patterns from the Existing Improved Weber Local Descriptor Let us consider an image with $3 \times 3$ matrix. Let $X_{c}$ be the centre pixel of a coral image as shown in Figure 2. Weber difference is calculated along $\mathrm{x}$ and $\mathrm{y}$ directions. Weber difference along $\mathrm{x}$ direction is estimated as shown in Eq. (1).

$$
\delta_{M-X}\left(X_{c}\right)=\arctan \left(\sum_{i=0}^{c_{i}-1} \frac{x_{i}-X_{c}}{x_{c}} \sin \theta_{i}\right)
$$

Weber Difference along y direction is estimated as shown in Eq. (2).

$$
\delta_{M-Y}\left(X_{c}\right)=\arctan \left(\sum_{i=0}^{c_{i}-1} \frac{X_{i}-X_{e}}{X_{c}} \sin \theta_{i}\right)
$$

Eq. (1) and Eq. (2) represents the angles between $\mathrm{x}$ direction and y direction. Here, $X_{c}$ represents the centre pixel and $X_{i}$ represents the neighboring pixels. The differential excitation occurs here is the relative intensity difference between the current pixels with its neighbors. The Weber magnitude is represented in Eq. (3) using Eq. (1) and Eq. (2).

$$
\delta_{M}\left(X_{c}\right)=\sqrt{\delta_{M-X}}\left(X_{c}\right)^{2}+\sqrt{\delta_{M-Y}}\left(X_{c}\right)^{2}
$$


The magnitude values obtained from these existing IWLD feature is the composition of the RGB channels. In the proposed method, Weber's magnitude is derived from each channel separately.

\subsection{Local Inter-Cross Weber Magnitude Patterns}

Let us consider the three kinds of improved Weber magnitude patterns. The channels are represented as Improved Weber Magnitude Red (IWMr), Improved Weber Magnitude Green (IWMg) and Improved Weber Magnitude Blue (IWMb) as depicted in the Figure 3. In Figure 3, Rc, Gc, Bc represents the centre pixel values. Then, red channel neighboring pixels are represented as R1, R2, R3, R4, R5, R6, R7, R8. Green channel neighboring pixels are represented as G1, G2, G3, G4, G5, G6, G7, G8. Blue channel neighboring pixels are represented as B1, B2, B3, B4, B5, B6, B7, B8.

The magnitude patterns of Weber Magnitude of each channel, namely Red, Green and Blue are derived as LICWMPrg, LICWMPrb, LICWMPgb. First, the magnitude pattern LICWMPrg can be obtained by first comparing the centre pixel value Rc with an improved Weber magnitude. Then, compared with its four red channel neighbors, namely R2, R4, R5, R7. Finally, a four bit code is generated by comparing its centre pixel and its neighbors. If the centre pixel value is greater than the neighboring pixel value then the value of binary code will be 1 or else 0 . Thus, binary code values are estimated using Eq. (4).

$$
\operatorname{LICWMP}_{\mathrm{rg}}(\mathrm{Rc})= \begin{cases}1, \text { if } N\left(x_{i}\right) \geq(R c) \\ 0, & \text { else }\end{cases}
$$
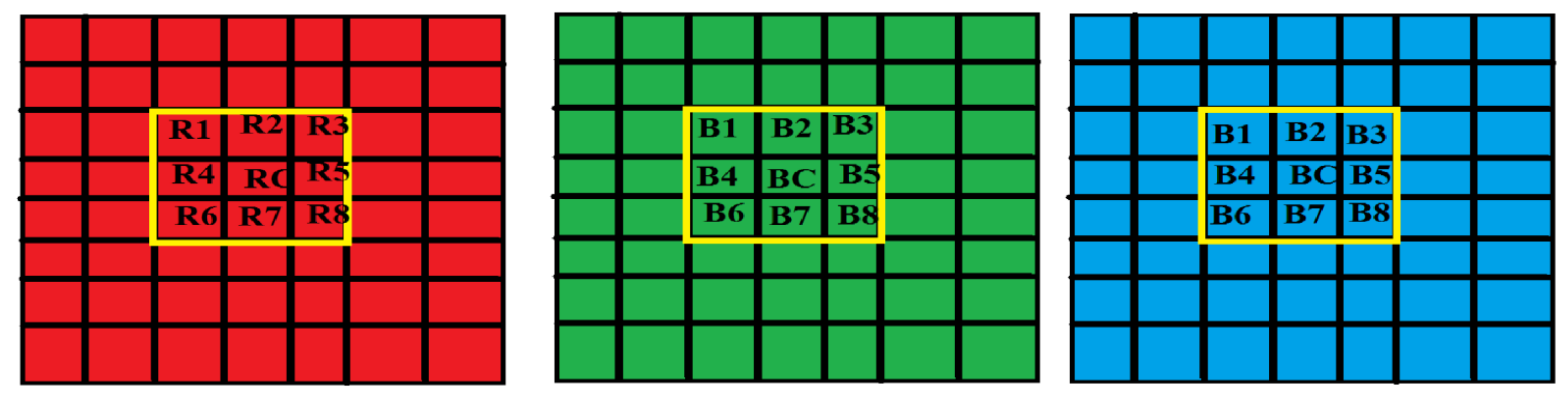

Figure 3 Representations of $\mathrm{IWM}_{\mathrm{r}}, \mathrm{IWM}_{\mathrm{g}}, \mathrm{IWM}_{\mathrm{b}}$

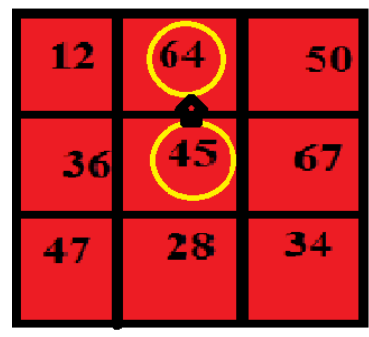

(a)

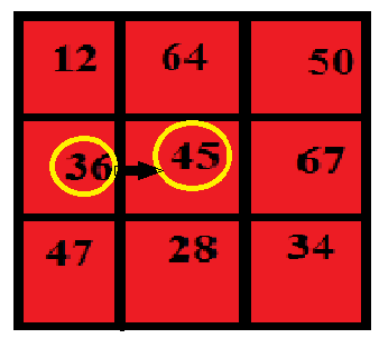

(c)

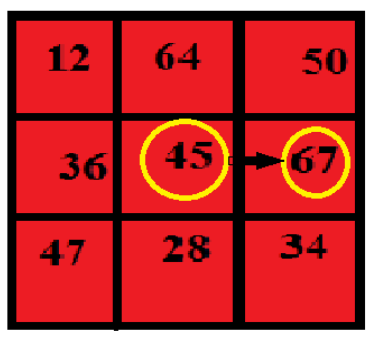

(b)

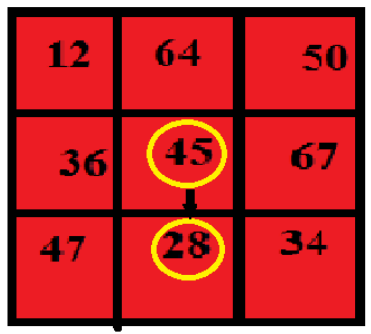

(d)

Figure 4 Illustration of Binary code for IWM $_{r}$

Next, in this Improved Weber Magnitude Red patterns, centre pixel Rc is compared with the corners of the green magnitude to obtain a binary code. The centre pixel Rc is compared with the corners of the green magnitude of G1, G3, G6, G7. By concatenating these binary codes in anticlockwise direction, the decimal values can be obtained from red and green magnitudes. Using these magnitude values, histogram can be generated. Similarly, all red magnitude values of centre pixel is again compared with the neighbors of Local red magnitude and Inter cross green magnitude to obtain the features of coral reef image. Using these intensity values, histogram is generated. The corresponding process is illustrated by the following steps. 

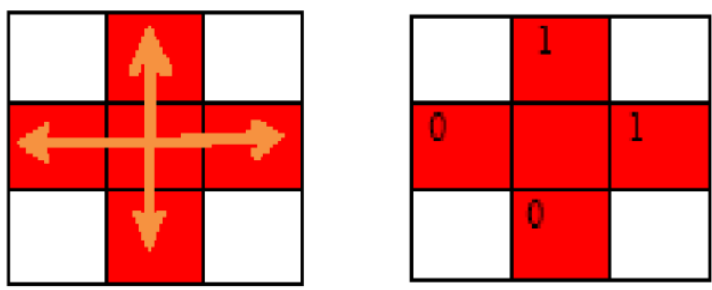

Figure 5 Binary code values generation

Step1: Binary codes are illustrated using Improved Weber magnitude red (IWMr)

As shown in the Figure 4, centre pixel Rc value is considered as 45. In IWMr, Rc value is first compared with its neighbor values. For example, here R2 pixel value is 64 compared as, $64>45$. Now the binary value of $\mathrm{R} 2$ is 1, because R2 value is greater than the centre pixel value. Likewise the steps are followed for all neighboring pixels. Finally the binary code values for the Local red magnitudes are generated as 1100 as shown in the Figure 5. The Figure 5 shows the corresponding binary code values for red magnitude.

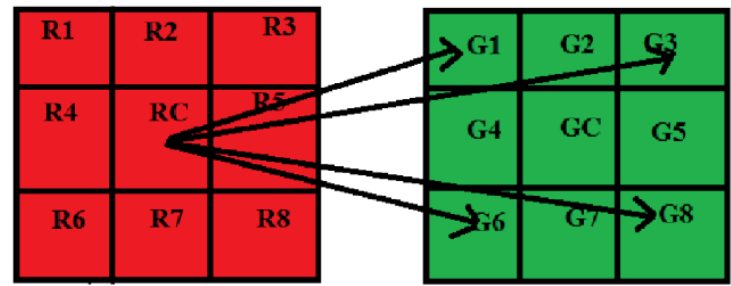

Figure 6 Comparison of Re with G1, G3, G6, G8

Step 2: Illustration of Binary code from IWMr and IWMg

In step 2 again the Red magnitude Rc is compared with green magnitudes, namely G1, G3, G6, G8 to get binary code as shown in the Figure 6. For example, the centre pixel Rc of red magnitude value is 45 , which is compared with the G1 of green magnitude with value 32 . Here $32<45$. Hence, the Binary code value will be 0 as shown in the Figure 7. Hence the steps are followed for other neighboring pixels, namely G3, G6 and G8 also.
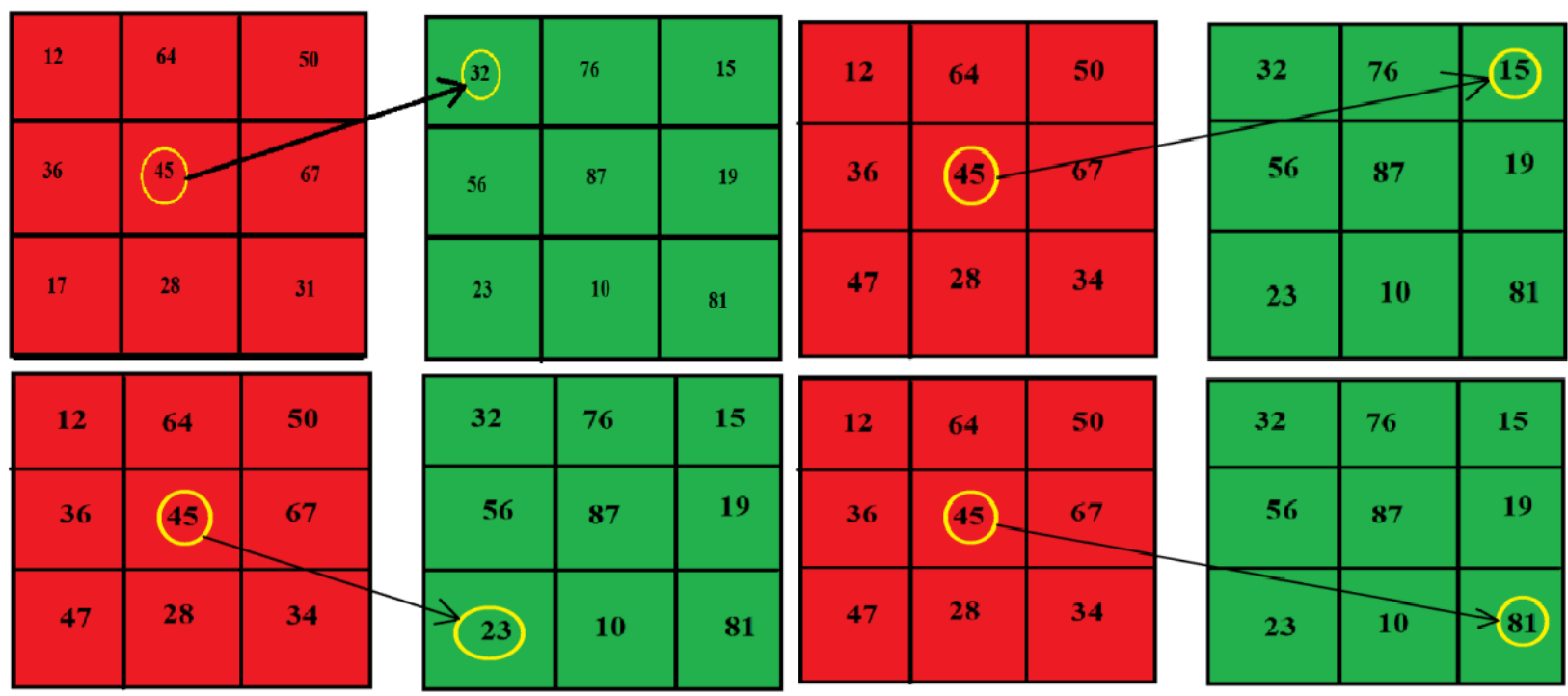

Figure 7 Comparison of Rc with G1, G3, G6, G8

Then, decimal values are generated using the binary codes as shown in the Figure 8. Decimal values are generated from binary codes by comparing red and green magnitudes as shown in the Figure 8 . The Binary codes which obtained from red and green magnitudes of step 1 and step 2 are concatenated in anticlockwise direction to get the binary code as shown in the Figure 9.

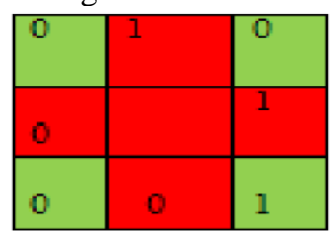

Figure 8 Binary code obtained 
This process is repeated for each and every position. Then, the histogram can be determined for LICWMPrg. Similarly, LICWMPrb is obtained by comparing red and blue magnitudes. LICWMPgb is also obtained by comparing green and blue magnitudes. Hence, the histogram is derived for this Local Inter-cross Weber Magnitude Pattern is used to recognise the features of coral reef images.
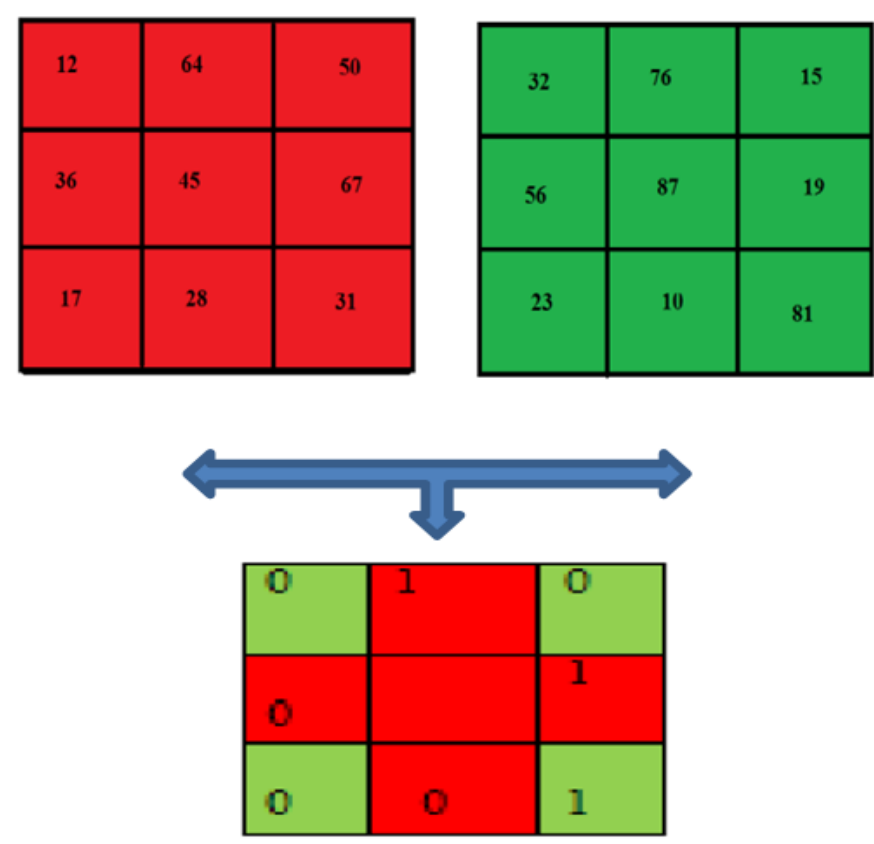

\subsection{VGG16 Architecture}

Figure 9 Encoding red and green magnitudes

VGG are commonly referred to as Deep learning network with Deep Layered architecture. It was developed by Visual Geometry Group at Oxford University. Deep features are extracted from raw input images by drifting to several hidden layers. VGG16 is the convolutional neural network architecture with 16 layers. The layers which are present in VGG16 are Convolutional layers, Max pooling layers, Activation Layers, and Fully Connected Layers. This architecture is the composition of 13 Convolutional layers, 5 max pooling layers and 3 dense layers which sums to form 21 layers but only have 16 weight layers.

In Conv1, 64 filters are used. Conv2 has 128 filters. Then Conv4 and Conv5 have utilized 512 filters. The convolution layer is the first layer which takes the input image and applies convolution filter to produce output which is fed to the successive layers. Here, multiple convolution layers are used for sub sampling to provide better overview.

In the proposed work, VGG implements deeper networks which give better extracting features in coral reef images. It is also able to distinguish one image from another class. It shows efficient performance. When fully connected layer is detached from the architecture it can be used to extract features of coral reef images. Again these deep features are classified using the classifiers KNN and Random Forest. The overview of VGG16 architecture is shown in the Figure 10. 


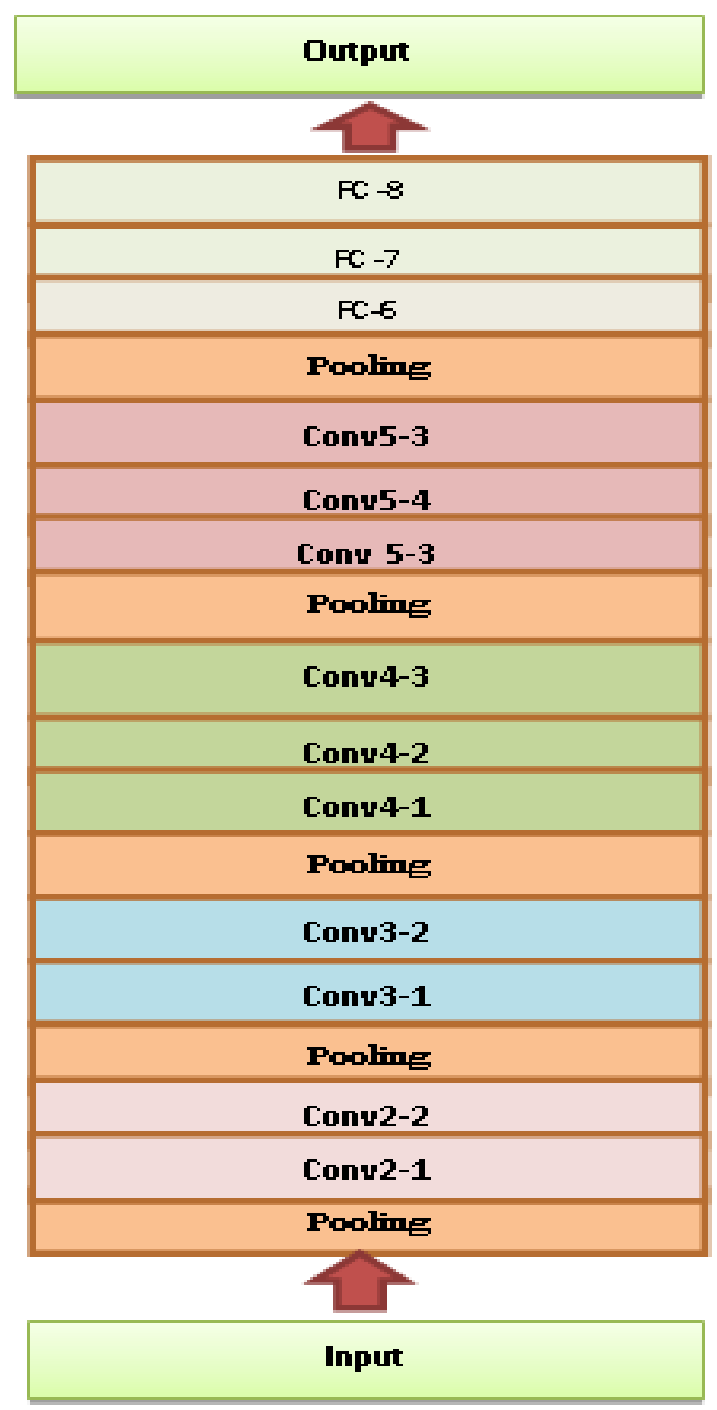

\subsection{Classifiers}

Figure 10 Architecture of VGG - 16

The process of grouping the tested image samples in to its particular classes often termed as texture classification. Each class is related according to its similarity features. The main aim of the classification algorithm is to group the images to its appropriate category. The image classification can be undergone by several classifiers. The classifiers used in the proposed system are, namely K-Nearest Neighbors (KNN) and Random Forest. 


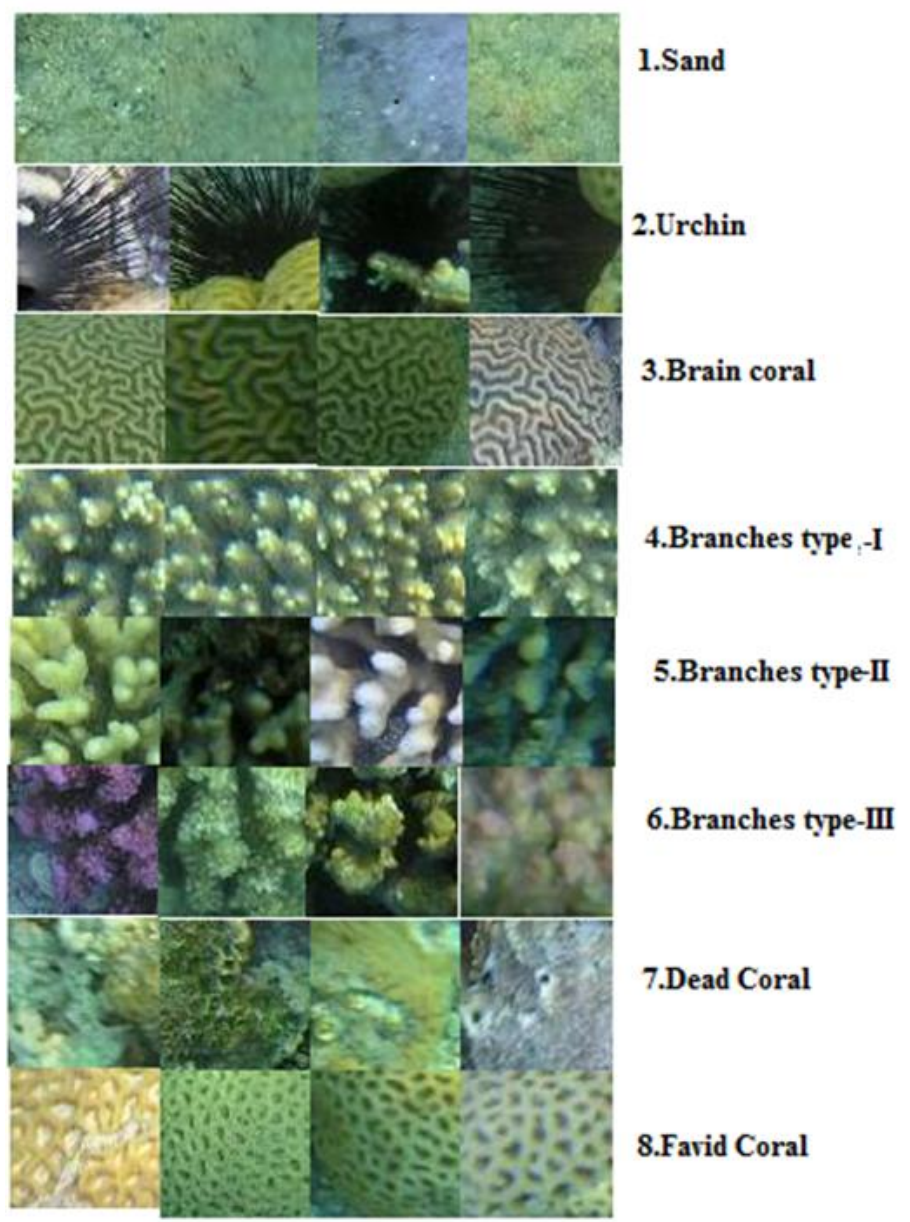

Figure 11 EILAT data set with samples

$\mathrm{K}$ - Nearest Neighbors (KNN) is a machine learning algorithm used in many applications. The main purpose of KNN is to classify the articular samples to its relevant classes according to their similarity measures. $\mathrm{KNN}$ classifier is used for classification [23] with Euclidean distance as the distance metric is given in Eq. (5).

$$
\left.\mathrm{KNN}(\mathrm{x}, \mathrm{u})=\sqrt{\left(x_{1}\right.}-u_{1}\right)^{2}+\cdots\left(x_{n}-u_{n}\right)^{2}
$$

Where $x_{n}, u_{n}$ represents the feature vectors.

Random Forest is one of the supervised learning algorithms with the collection of several decision trees. It is the most popular model with better results. The best possible results are arrived by generating random uncorrelated decision tree. 


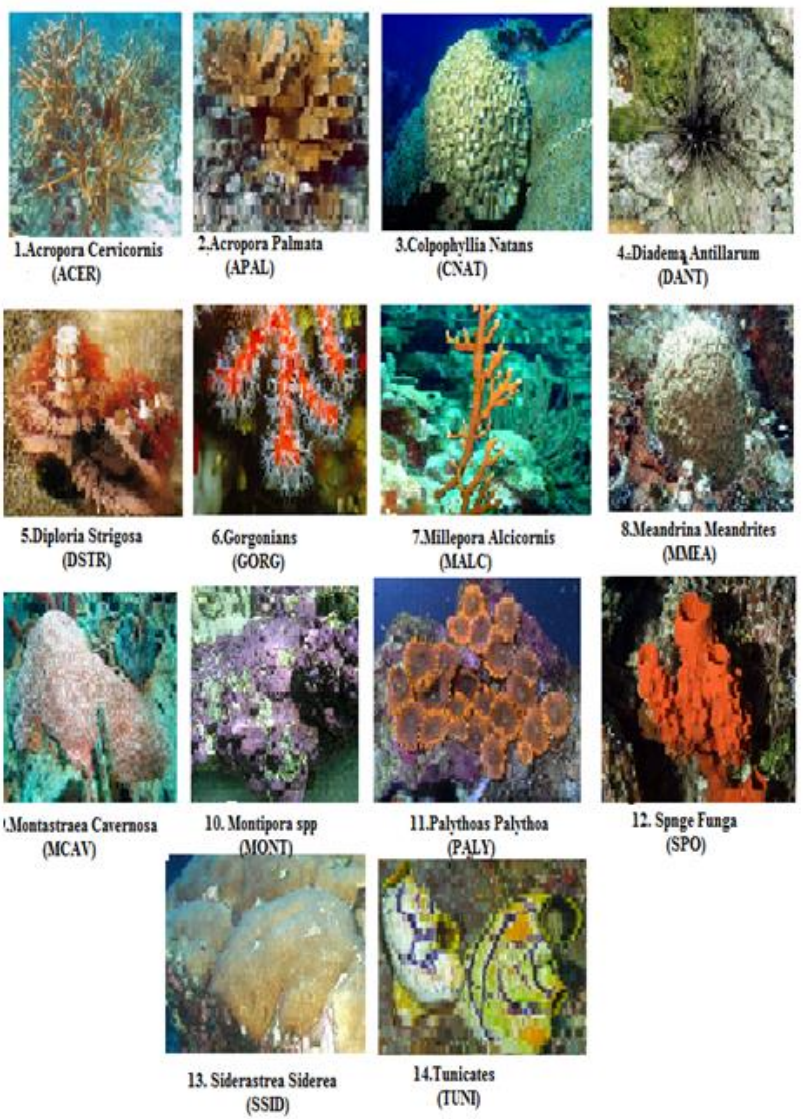

4 Experimental Results and Analysis

Figure 12 RSMAS data set with coral names and sample images

\subsection{Data set Description}

EILAT data set is used in the proposed system with 1123 image samples. The particular coral species are identified by its labels. Eight classes are found in EILAT data set. The eight class coral names are, namely Branches type III, branches type II, branches type I, brain coral, urchin, favid coral, dead coral and sand. The resolution size of the images in this data set is $64 \times 64$. The sample images are represented in the Figure 11 .

The image patches in RSMAS data set are collected from Rosensteil School of marine and Atmospheric Science of University Miami. The size of the image patches are 256x 256. The images are collected from different places using different cameras. These image patches are classified in to 14 image patches with their class names. The coral class names of RSMAS data set are Tunicates, Acropora palmata, Sponge fungus, Colpophyllia natans, Diploria strigosa, Siderastrea siderea, Gorgonians, Palythoas palythoa, Millepora alcicornis, Montipora spp., Montastraea cavernosa, Diadema antillarum, Meandrina meandrites and Acropora cervicornis. The sample images are represented in the Figure 12.

\subsection{Performance Metrics}

Performance metrics are used to estimate the classification models used in the proposed

system.

Experimentations are accompanied with the data set EILAT. Performance metrics used in this proposed work are Accuracy, Sensitivity, Specificity, Precision, F1Score and Recall. The procedures used to evaluate the above performances are True Positive (TP), False Negative (FN), True Negative (TN) and False Positive (FP).

True Positive (TP) is used to monitor the results as positive and the anticipated result as positive. False Negative (FN) is used to monitor the results as positive but the anticipated result as negative. True Negative (TN) is used to monitor the results as negative and the anticipated result as negative. False Positive (FP) is used to monitor the results as negative but the anticipated result as positive.

Accuracy [24] is defined as a measured value is to the actual (true) value. It is the ratio of the number of correct analysis to the total number of input samples as shown in Eq. (6).

$$
\text { Accuracy }=\frac{\text { Number of Accurate Predictions }}{\text { Total number of Predictions }}
$$

Sensitivity [24] is defined as the True Positive Rate (TPR) relays to the fraction of positive data points that are correctly measured as positive with respect to all positive data points as shown in Eq. (7). 


$$
\text { Sensitivity }=\frac{T P}{T P+F N}
$$

Specificity [24] is defined as the False Positive Rate (FPR), which corresponds to the fraction of negative data points that are fallaciously considered as positive, with respect to all negative data points as shown in Eq. (8).

$$
\text { Specificity }=\frac{T N}{F P+T N}
$$

F1 score is used to measure the test precision. It is the Mean of precision and recall. It is shown in Eq. (9). Here, Precision is defined as the total correct positive results divided by the number of positive results anticipated by the classifier.

$$
\text { F1 Score }=2 * \frac{\text { recall } * \text { precision }}{\text { recall }+ \text { Precision }}
$$

\subsection{Classification Results}

The Experimental results are summarised in Table 1. Table 1 shows how effectively the coral images are classified with Local descriptors and deep learning methods. The first three rows display the results conducted with LAP, LBP and IWBC. Table 1 proves that IWBC is the best local descriptors for feature extraction with KNN classifier from previous work [21]. In this proposed work the novel operator LIWMP achieve better results compared with the existing IWBC.

First the experiment is conducted with the handcrafted methods and the results are analysed and displayed. Table1 shows the result which was obtained by EILAT data set. The next experiment is conducted with VGG-16 deep network architecture which shows the best classification results. For KNN classifier, this proposed method obtained $92.95 \%$ sensitivity, 97.42\% precision and 94.25\% F1score. For Random Forest

\begin{tabular}{|c|c|c|c|c|c|c|}
\hline Methods & \multicolumn{2}{|c|}{ Sensitivity } & \multicolumn{2}{|c|}{ Precision } & \multicolumn{2}{|c|}{ F score } \\
\hline Classifier & KNN & $\mathbf{R F}$ & KNN & $\mathbf{R F}$ & KNN & $\mathbf{R F}$ \\
\hline LBP & 95.9 & 64.2 & 76 & 82.2 & 75.9 & 67.36 \\
\hline LAP & 69.8 & 60 & 64.8 & 75.9 & 66.43 & 63.77 \\
\hline IWBC & 88.7 & 73.6 & 87 & 88.5 & 87.5 & 64.7 \\
\hline VGG & 92.95 & 91.55 & 97.42 & 97.12 & 95.13 & 94.25 \\
\hline VGG+Proposed & 96.35 & 94.5 & 97.67 & 97.25 & 94.64 & 94.87 \\
\hline Proposed & 90.57 & 90.05 & 95.78 & 91.22 & 93.1 & 90.6 \\
\hline
\end{tabular}
classifier, this proposed method obtained $95.13 \%$ sensitivity, $91.55 \%$ precision and $97.12 \%$ F1score. The results clearly states that KNN classifier shows better results compared to Random Forest classifier.

Table 1: Sensitivity,Precision,FScore of various Descriptors using EILAT Dataset

Table 2 represents the results conducted with hydrid methods. The experiments are also performed with the hybrid methods of VGG and the proposed operator LICWM Pattern. For RSMAS data set, KNN classifier provides sensitivity value of $96.35 \%$, precision value of $97.67 \%$ and F1score value of $94.64 \%$. For random forest classifier, $94.5 \%$ is obtained for sensitivity, $97.25 \%$ is obtained for precision and $94.87 \%$ is obtained for F1score. The results with the proposed operator depicts that the sensitivity value is $90.57 \%$, precision value is $95.78 \%$ and $\mathrm{F} 1$ score value is $93.1 \%$ for $\mathrm{KNN}$ classifier. The results with the proposed operator depicts that the sensitivity value is $90.05 \%$, precision value is $91.22 \%$ and F1score value is $90.6 \%$ for random forest classifier.

\begin{tabular}{|l|l|l|l|l|l|l|}
\hline \multicolumn{1}{|c|}{ Methods } & \multicolumn{2}{c|}{ Sensitivity } & \multicolumn{2}{c|}{ Precision } & \multicolumn{2}{c|}{ F score } \\
\hline Classifier & KNN & RF & KNN & RF & KNN & RF \\
\hline LBP & 73.59 & 61.33 & 75.59 & 71.11 & 74.57 & 67.52 \\
\hline LAP & & & & & & \\
\hline
\end{tabular}




\begin{tabular}{|l|l|l|l|l|l|l|}
\hline IWBC & 85.9 & 77.6 & 86.6 & 83.9 & 86.2 & 80.62 \\
\hline VGG & 90.59 & 88.74 & 91.33 & 90.32 & 90.85 & 89.52 \\
\hline VGG+Proposed & 91.8 & 90.66 & 93.02 & 92.02 & 92.48 & 91.33 \\
\hline Proposed & & & & & & \\
\hline
\end{tabular}

Table 2: Sensitivity,Precision,FScore of various Descriptors using RSMAS Dataset

By comparing all the three results, IWBC acquire best results than LAP and LBP with Fscore $86.2 \%$ for KNN and 80.62 for Random Forest. Next, the experiments are conducted with deep learning method. VGG - 16 architecture is applied for experimental analysis and for classification, KNN classifier and Random Forest classifier is used. For KNN classifier, sensitivity obtained is $90.59 \%$, precision obtained is $91.13 \%$ and F1score obtained is $90.85 \%$. For random forest classifier, sensitivity obtained is $88.74 \%$, precision obtained is $90.32 \%$ and $\mathrm{F} 1$ score obtained is $89.52 \%$.

The results are taken when deep learning method is fused with the proposed operator LIWMP and applied in EILAT data set. This provides 91.8\% sensitivity, 93.02\% precision and 92.48\% F1score for KNN classifier. Random forest classifier provides $90.66 \%$ sensitivity, $92.2 \%$ precision and $92.48 \%$ F1score. The proposed operator LIWMP is also tested for RSMAS data set. The results show $90.08 \%$ for sensitivity, $90.25 \%$ for precision and $90.16 \%$ for F1scoreby classifying using KNN classifier. The results show $90.16 \%$ for sensitivity, $91.2 \%$ for precision and $90.67 \%$ for F1scoreby classifying using random forest classifier. Among all these results, the proposed operator with deep learning method achieves better results than other feature descriptors for both data sets.

Table 3 depicts the results of the proposed method LICWMP compared with the existing Descriptors, namely CLBP based method by Mohammad [15]. Ani Brown Mary et al [17], [18] implemented ILDP and Z ILBP. Padma [22] has implemented VGG -16 Deep learning methods for classification are also listed in the Table 3. Among all these existing works displayed below the proposed operator with VGG -16 achieve better performance with than other descriptors. The proposed method is used for extracting the features of coral reef images. KNN and Random Forest is used as classifier. The proposed method achieves high accuracy than the existing operators. In the proposed work, the Intercross method is used for feature extraction which covers all directions of pixels from centre pixels.

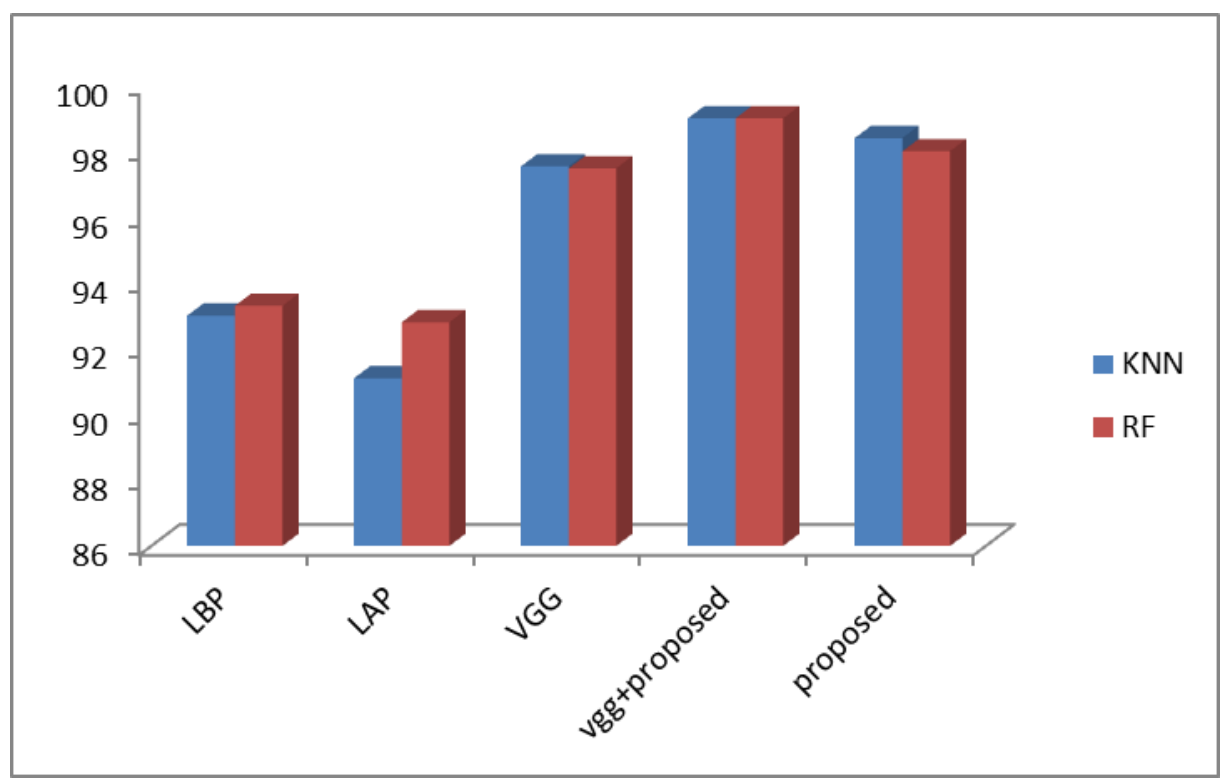

Graph1 The proposed operator Accuracy with various Descriptor 


\begin{tabular}{|c|c|c|c|c|}
\hline Author & Techniques & Classifier & Eilat Dataset & RSMAS Dataset \\
\hline Mohammad[15] et al.(2017) & CLBP & KNN & 88.3 & 83.5 \\
\hline ANI[18] et al. (2017) & ILDP & SVM,CNN,KNN & 97.5 & 97.1 \\
\hline ANI[18] et al. (2017) & ZtTZLBP & KNN & 97.3 & 97.8 \\
\hline \multirow{3}{*}{ Padma[21] et al. (2019) } & LBP & $\begin{array}{l}\text { KNN } \\
\text { Random Forest }\end{array}$ & $\begin{array}{l}93.2 \\
93.3\end{array}$ & $\begin{array}{c}96 \\
93.9\end{array}$ \\
\hline & LAP & $\begin{array}{l}\text { KNN } \\
\text { Random Forest }\end{array}$ & $\begin{array}{l}91.1 \\
92.8\end{array}$ & $\begin{array}{l}94.6 \\
94.6\end{array}$ \\
\hline & IWBC & $\begin{array}{l}\text { KNN } \\
\text { Random Forest }\end{array}$ & $\begin{array}{l}97.4 \\
94.2\end{array}$ & $\begin{array}{l}98.1 \\
96.8\end{array}$ \\
\hline Padma[22] et al. (2020) & VGG-16 & $\begin{array}{l}\text { KNN } \\
\text { Random Forest }\end{array}$ & $\begin{array}{l}97.5 \\
97.4\end{array}$ & $\begin{array}{l}98.7 \\
98.4\end{array}$ \\
\hline Proposed + VGG & $\begin{array}{l}\text { LICWMP + } \\
\text { VGG }\end{array}$ & $\begin{array}{l}\text { KNN } \\
\text { Random Forest }\end{array}$ & $\begin{array}{l}98.8 \\
98.6\end{array}$ & $\begin{array}{l}98.8 \\
98.6\end{array}$ \\
\hline Proposed & LICWMP & KNN & 98.3 & 99 \\
\hline
\end{tabular}

Table 3 The overall accuracy of the proposed operator with various descriptor

\section{CONCLUSION}

A novel operator called Local Inter Cross Weber Magnitude pattern has been proposed for feature extraction and classification of coral reef images. Experiments has been conducted with the proposed operator are integrated with deep features to improve better performance. Classifiers, namely KNN and Random forest are applied effectively for classification. On comparing the results with LICWMP the proposed operator, the proposed operator provides better results. The proposed method also provides efficient results on comparing with the other traditional local descriptors, namely LAP, LBP, IWBC, CLBP and ILDP. When the proposed method is integrated with VGG-16, its performance has been improved to 98.8\%. These Experiments are conducted with EILAT data set and RSMAS data set. This proposed operator LICWMP can also be integrated with various machine learning algorithms for further research.

\section{REFERENCES}

Jie Chen,"WLD : A Robust Local Image Descriptor", IEEE Transactions on Pattern Analysis and Machine Intelligence, Vol 32, No.9, September 2010.

Joshua V.Stough "Texture and Color Distribution-based Classification for Live Coral Detection ", Proceedings of the $12^{\text {th }}$ International Coral Reef Symbosium,Cairns,Australia, 9-13 July 2012 5D Robots and Machine learning techniques aiding coral reef science.

Hassan Dawood,'Texture Image Classification with Improved Weber Local Descriptor" ICAISC 2014, pp. 684692,Springer International "Publishing Switzerland 2014.

Deepshika Tiwari,"Improved Weber's law based local binary pattern for dynamic texture recognition", Springer Science + Business Media New York 2016. 
D.G.Agrawal,'Dynamic Texture Feature Extraction Using Weber Local Descriptor", International Journal of Engineering Research and Applications, Vol.4, pp.502-506 March 2014.

Amit Satpathy,'LBP - Based Edge Texture Features for Object Recognition",IEEE Transactions on Image Processing,May 2014.

Jinwang Feng,"Dominant - Completed Local Binary Patterns for Texture Classification", International Conference on Information and Automation Lijiang,China,August 2015.

Zhenhua Guo," A Completed Modeling of Local Binary Pattern Operator for Texture Classification. IEEE Transactions on Image Processing,Vol 19,No.6,June 2010.

Soo - Chang Pei, "Compact LBP and WLBP Descriptor with magnitude and Direction Difference for face Recognition”,IEEE 2017.

R.Ahilapriyadharshini,"Weber Local Descriptor based Object Recognition", International Conference on Advanced Communication Control and Computing Technologies 2012.

Fan Liu,’WLBP: Weber Local Binary Pattern for Local Image Descriptor",NeuroComputing 2012.

Zuodong Yang,"Weber Binary Pattern and Weber Ternary Pattern for Illumination Robust Face Recognition",Proceedings of APSIPA Annual Summit and Conference 2015.

Thanh Phuong Ngugen,"Statistical binary patterns for rotational invariant texture classification", Neurocomputing,2016.

Ngoc-Son Vu, "Exploring Patterns of Gradient Orientations and Magnitudes for Face Recognition" IEEE Transactions on Information Forensics and Security,Vol 8,No.2,February 2013295.

Mohammad Hossein Shakoori, "A novel advanced local binary pattern for image-based coral reef classification",Springer Science + Media New York 2017.

Ani Brown Mary, "Coral reef image/video classification employing novel octa-angled pattern for triangular sub region and pulse coupled convolutional neural network(PCCNN)"Springer 2018.

N.Ani Brown Mary "Classification of coral reef submarine images and Videos using a Novel Z with Tilted Z Local Binary Pattern( $\mathrm{Z}^{\oplus}$ TZLBP)"Springer 2017.

N. Ani Brown Mary,Dejey Dharma "Coral reef image classification employing Improved LDP for feature Extraction" Journal of visual communication and image representation,2017

R.Hatibaruah, "Elliptical local binary co-occurrence pattern for face image retrieval”, Journal of Statistics and Management System 2019.

N.Ani Brown Mary," A novel framework for real time diseased coral reef image classification"Springer 2018.

C.Padma Priya,"Coral Reef Image Classification with Hybrid Methods",International Journal of Innovative Technology and Exploring Engineering ,2019.

C.Padma Priya,’Deep Features Based Coral Reef Classifications using Machine Learning.,"JETIR ,2020

N. Ani Brown Mary, A. Robert Singh, and Suganya Athisayamani, "Classification of Banana Leaf Diseases Using Enhanced Gabor Feature Descriptor”, Inventive Communication and Computational Technologies, Proceedings of ICICCT 2020.

K. Jayapriya, N. Ani Brown Mary, "Employing a novel 2-gram subgroup intra pattern (2GSIP) with stacked auto encoder for membrane protein classification", Springer, Molecular Biology Reports, https://doi.org/10.1007/s11033-019-04680-3, 2019. 